\title{
Optimum LAI for Yield Maximisation of Finger Millet under Irrigated Conditions
}

\author{
Mujahid Anjum, Y. A. Nanja Reddy* and M. S. Sheshshayee \\ Department of Crop Physiology, University of Agricultural Sciences, \\ Bengaluru-560065, Karnataka, India \\ *Corresponding author
}

\section{A B S T R A C T}

\begin{tabular}{l} 
Key w o r d s \\
$\begin{array}{l}\text { Plant density, leaf } \\
\text { area, photosynthetic } \\
\text { rate, productive } \\
\text { tillers, grain yield }\end{array}$ \\
\hline Article Info \\
\hline $\begin{array}{l}\text { Accepted: } \\
\text { 10April 2020 } \\
\text { Available Online: } \\
\text { 10 May 2020 }\end{array}$ \\
\hline
\end{tabular}

\section{Keywords}

Plant density, leaf area, photosynthetic te, productive

Article Info

Accepted: Available Online 10 May 2020
Field experiment was conducted during summer, 2018 to determine the influence of LAI on yield maximisation in finger millet genotypes by varying plant densities. Maximum grain yield was obtained at the plant density of 44.4 to 66.6 hills $\mathrm{m}^{-2}$ but above or below. The source size (LAI) and source activity (photosynthetic rate) were not the limitations for yield maximisation under optimal irrigation and; LAI of 6.5 to 7.0 was optimum for maximum finger millet yield especially in variety, GPU-28. The sink traits, namely productive tillers per $\mathrm{m}^{-2}$ and mean ear weight were compensated to each other $(\mathrm{r}=-0.967 * * *)$. The plant density of 44.4 hills $\mathrm{m}^{-2}(22.5 \mathrm{~cm} \times 10 \mathrm{~cm})$ could be optimum for irrigated finger millet. Further yield enhancement could be possible by increasing productive tillers (up to 5.0 per hill) with plant density of 44.4 hills $\mathrm{m}^{-2}$ varying spacing to $30.0 \mathrm{~cm} \times 7.5 \mathrm{~cm}$.

\section{Introduction}

Finger millet is a $\mathrm{C}_{4}$ crop belongs to family poaceae (Dida et al., 2007) cultivated in arid and semi-arid regions in more than 25 countries. In India as a staple food and fodder crop, it is cultivated an area of 1.19 million hectares with a production of 1.98 lakh tones and productivity of $1661 \mathrm{~kg} \mathrm{ha}^{-1}$, Karnataka being the major producer to the extent of 58 per cent (Anon., 2015; Sakamma et al., 2018).
Although finger millet is cultivated as rainfed crop by more than $90 \%$ area (Davis et al., 2019), crop being responsive to irrigation and external fertilizer application (Gull et al., 2014; Thilakarathna and Raizada, 2015; Ramakrishnan et al., 2017; Wafula et al., 2018), it is cultivated during summer season wherever irrigation facilities are available. Finger millet is highly nutritious crop with its composition of protein $(7.3 \%)$, fat $(1.3 \%)$, carbohydrates $(72.6 \%)$, dietary fibre $(18 \%)$, 
Ash (3.0\%), calcium (352mg/100g) and Leucine, $594 \mathrm{mgg}^{-1}$ of protein (Shobana et al., 2013; Devi et al., 2014; Chandra et al., 2016; Gupta et al., 2017; Sharma et al., 2017). In addition, it has high soluble fibre, polyphenols coupled with high resistant starch, thus slow hydrolysis of starch and; gaining importance with increasing diabetic population (Kumari and Sumathi, 2002).

For yield improvement of finger millet, early research efforts were made to select large ear size as the tiller number was not a constraint (8.0 tillers hill $^{-1}$ in popular varieties at that time, Krishnamurthy, 1971). Probably, selection for ear size with time, the tiller numbers might have compensated with ear size and resulted in selection of shy tillering genotypes. It is clearly evident in the popular variety GPU-28 which has only 2 to 2.5 tillers hill $^{-1}$ with a mean ear weight of 6.0 to $7.0 \mathrm{~g}$ (Prakasha et al., 2018). In recent years, it was observed that the major yield attributes in finger millet are the productive tillers (contributes to $54 \%$ of yield), followed by ear weight and test weight although it is genotypic character (Anon., 2015). Increase in productive tillers per unit land area can be achieved by manipulating the population density (Richards, 2000). Therefore, additional productive tiller per hill could enhance the potential yield of GPU-28.

Formation of productive tillers and consequent grain yield of finger millet are determined by the source size and activity. The source size in finger millet is not a major limitation as a cereal crop (Patrick, 1988) and the photosynthetic rate is also relatively high being a $\mathrm{C}_{4}$ species (Berdahl et al., 1971; Ueno et al., 2006). Hence, tiller production is an important sink trait in determining the grain yield which can be addressed though manipulating the planting density under adequate irrigation and soil fertility. Therefore, the optimum source size (LAI) and productive tillers required for maximum grain yield in finger millet was investigated with varying plant densities.

\section{Materials and Methods}

The experiment was conducted during summer, 2018. Three finger millet genotypes (GE-292, GE-199 and GPU-28) were evaluated in factorial RCBD comprising of seven spacing treatments (given with data) in four replications. Experiment was conducted at the Field Unit, Department of Crop Physiology, Zonal Agricultural Research Station, GKVK, University of Agricultural Sciences, Bengaluru-65. The finger millet genotypes were sown on 12/01/2018 in plastic portrays and 17 days old seedlings were transplanted in the main field $(29 / 01 / 2018)$ in five rows of 1.2 meter length with respective spacings as per the treatments.

At the time of flowering, observations on leaf area, light penetration, chlorosis of older leaves and photosynthetic rate were measured. The $3^{\text {rd }}$ leaf area (length $\mathrm{x}$ width $\mathrm{x}$ 0.75) was multiplied by total number of leaves in all the tillers in a hill to arrive at leaf area per plant. The leaf area index (LAI) was computed by dividing the total leaf area with the spacing per hill according to the treatments. The light insolation at ground level (light penetrated to the ground) was recorded by placing the light quantum sensor (Li-Cor) between the rows. The number of basal leaves turned yellow (more than half part of the leaf becomes chlorotic) on the main tiller was counted at 20 days after anthesis. The photosynthetic rate was measured using Infrared Gas Analyser (IRGA) (Cyrus) from 9.00 to $11.00 \mathrm{AM}$ on $20^{\text {th }}$ day after flowering. The yield attributes viz., productive tillers, mean ear weight and test weight were measured at the time of harvest. All these measurements were made in net plot area of three rows of 1.0 meter row 
length and computed to per square meter area. The spikelet fertility was calculated by cutting $2 \mathrm{~cm}$ finger length and carefully counted the number of florets and seeds. The fertility was then calculated as the number of filled grains / total number of florets multiplied by 100 . The data was statistically analysed in factorial RBD using OPSTAT (Sheoran et al., 1998).

\section{Results and Discussion}

Early efforts on yield improvement of finger millet were basically through selection for large ear size, wherein productive tillers per hill was not a constraint (Krishnamurthy, 1971). Next stage of improvement was through plant breeding efforts for blast resistance combined with adoption of improved management practices. In recent years, finger millet yield has reached a plateau (Swetha, 2011). Among the cultivated varieties, most popular variety GPU-28 is a shy tillering type with relatively a large ear size (Prakasha et al., 2018). Therefore, the plant density was altered to increase the leaf area, productive tillers and consequent grain yield of finger millet.

The plant density of $44.4 \mathrm{~m}^{-2}$ (recommended spacing of $22.5 \mathrm{~cm} \times 10 \mathrm{~cm}$ ) resulted in higher grain yield of $737.7 \mathrm{~g} \mathrm{~m}^{-2}$ over the plant density of $33.3 \mathrm{~m}^{-2}\left(645.2 \mathrm{~g} \mathrm{~m}^{-2}\right)$ and $22.2 \mathrm{~m}^{-2}$ $\left(613.0 \mathrm{~g} \mathrm{~m}^{-2}\right)$. The higher plant density $(66.7$ $\mathrm{m}^{-2}$ ) and more did not increase the grain yield significantly (Table 1). Similarly, increase in row spacing from 20 to $30 \mathrm{~cm}$ (Bitew and Asargew, 2014; Dereje et al., 2016) and row spacing up to $45 \mathrm{~cm}$ (Yoseph, 2014) have increased the grain yield significantly; with no significant differences between 30 and 45 $\mathrm{cm}$ row spacing (Yoseph, 2014). The plant density with higher spacing of $45 \mathrm{~cm}$ and above between the rows decreased the grain yield due to reduced number of tillers per unit area (Bitew and Asargew, 2014; Dereje et al., 2016). Therefore, the optimum spacing could be between 20 to $30 \mathrm{~cm}$ between rows and 7.5 to $10 \mathrm{~cm}$ between the plants. The increased grain yield was due to increased total biomass production $\left(\mathrm{r}=0.457^{*}\right.$, Table 2$)$ with no influence of harvest index (HI) as HI did not differ between treatments (Table 1). Similarly significant positive association between biomass and grain yield has been reported (Negi et al., 2017; Prakasha et al., 2018; Nanja Reddy et al., 2019; Chavan et al., 2020; Somashekhar and Loganandhan, 2020). Such biomass production will be determined by the LAI and photosynthetic rate.

The LAI (source size) showed a positive significant relationship with biomass $(\mathrm{r}=$ $\left.0.803^{* *}\right)$, productive tillers $\left(\mathrm{r}=0.687^{* *}\right)$ and grain yield $\left(r=0.528^{*}\right)$ (Table 2$)$. The mean grain yield was increased with an increase in LAI up to 7.0, beyond which the grain yield was decreased (Fig. 1a). Among the varieties, GPU-28 gave the grain yield of $685.3 \mathrm{~g} \mathrm{~m}^{-2}$ at the recommended spacing of $22.5 \mathrm{~cm} \mathrm{x} 10 \mathrm{~cm}$ (LAI of 7.96), while narrow spacing ( $15 \mathrm{~cm} \mathrm{x}$ $10 \mathrm{~cm}$ ) marginally increased the grain yield $\left(711.1 \mathrm{~g} \mathrm{~m}^{-2}\right.$, the LAI was 6.34$)$ and further increase in plant density (up to $200.0 \mathrm{~m}^{2}$ by $10 \mathrm{~cm} \times 5 \mathrm{~cm}$ ) did not result in higher grain yield significantly (Table 1).

These results imply that the optimum LAI for higher grain yield could be between 6.5 and 7.0 especially in case of variety, GPU-28. At plant density above $44.4 \mathrm{~m}^{-2}$, the light penetration to the ground level was decreased with an increased chlorosis of older leaves (Table 3). Probably, at narrow spacing with higher LAI, the microclimate has poor aeration and lead to higher maintenance respiration, and reduced grain yield by reducing the partitioning (harvest index). The wider spacing reduced the LAI significantly as compared to the recommended spacing $(22.5 \mathrm{~cm} \times 10 \mathrm{~cm})$, biomass production and grain yield. 
Table.1 Effect of plant densities on biomass, harvest index and grain yield in finger millet genotypes

\begin{tabular}{|c|c|c|c|c|c|c|c|c|c|c|c|c|c|}
\hline \multirow{2}{*}{$\begin{array}{l}\text { Spacing } \\
(\mathrm{cm} \times \mathrm{cm}) / \\
\text { Varieties }\end{array}$} & \multirow{2}{*}{$\begin{array}{c}\text { Plant } \\
\text { density } \\
(\text { No. } \\
\left.\text { m }^{-2}\right)\end{array}$} & \multicolumn{4}{|c|}{ Biomass $\left(\mathrm{g} \mathrm{m}^{-2}\right)$} & \multicolumn{4}{|c|}{ Harvest index } & \multicolumn{4}{|c|}{ Grain yield $\left(\mathrm{g} \mathrm{m}^{-2}\right)$} \\
\hline & & $\begin{array}{l}\text { GE- } \\
292\end{array}$ & $\begin{array}{l}\text { GE- } \\
199\end{array}$ & $\begin{array}{c}\text { GPU- } \\
28\end{array}$ & Mean & $\begin{array}{l}\text { GE- } \\
292\end{array}$ & $\begin{array}{c}\text { GE- } \\
199\end{array}$ & $\begin{array}{c}\text { GPU- } \\
28\end{array}$ & Mean & $\begin{array}{l}\text { GE- } \\
292\end{array}$ & $\begin{array}{c}\text { GE- } \\
199\end{array}$ & $\begin{array}{c}\text { GPU- } \\
28\end{array}$ & Mean \\
\hline$T_{1}(30 \times 15)$ & 22.2 & 1548 & 1534 & 2056 & 1713 & 0.36 & 0.41 & 0.32 & 0.36 & 556.0 & 623.2 & 662.6 & 613.9 \\
\hline$T_{2}(30 \times 10)$ & 33.3 & 1745 & 1598 & 2019 & 1788 & 0.41 & 0.40 & 0.29 & 0.37 & 718.7 & 635.7 & 581.2 & 645.2 \\
\hline$T_{3}(22.5 \times 10)$ & 44.4 & 1838 & 1932 & 2437 & 2069 & 0.41 & 0.41 & 0.28 & 0.36 & 745.2 & 782.7 & 685.3 & $\mathbf{7 3 7 . 7}$ \\
\hline$T_{4}(15 \times 10)$ & 66.7 & 2439 & 2036 & 2108 & 2194 & 0.32 & 0.38 & 0.34 & 0.34 & 776.3 & 776.5 & 711.1 & 754.7 \\
\hline$T_{5}(10 \times 10)$ & 100.0 & 2199 & 2123 & 2098 & 2140 & 0.30 & 0.37 & 0.32 & 0.33 & 661.5 & 774.1 & 665.6 & 700.4 \\
\hline$T_{6}(10 \times 7.5)$ & 133.3 & 2206 & 1790 & 2147 & 2048 & 0.33 & 0.36 & 0.33 & 0.34 & 737.2 & 648.9 & 717.6 & 701.2 \\
\hline$T_{7}(10 \times 5)$ & 200.0 & 2026 & 1879 & 2009 & 1971 & 0.36 & 0.40 & 0.37 & 0.38 & 730.8 & 746.1 & 750.6 & 742.5 \\
\hline Mean & & 2000 & 1842 & 2125 & & 0.36 & 0.39 & 0.32 & & 703.7 & 712.5 & 682.0 & \\
\hline Factors & & $\mathrm{SEm} \pm$ & $\begin{array}{l}\text { C.D } \\
@ \text { @ } 5 \%\end{array}$ & & & $\mathrm{SEm} \pm$ & $\begin{array}{c}\text { CD @ } \\
5 \%\end{array}$ & & & $\mathrm{SEm} \pm$ & $\begin{array}{c}\mathrm{CD} @ \\
5 \%\end{array}$ & & \\
\hline Treatments & & 59 & 170 & & & 0.01 & NS & & & 29.2 & 83.7 & & \\
\hline Genotypes & & 39 & 111 & & & 0.01 & 0.02 & & & 19.1 & NS & & \\
\hline Interaction & & 102 & 294 & & & 0.02 & 0.06 & & & 50.5 & NS & & \\
\hline C.V (5\%) & & 8.9 & & & & 8.8 & & & & 12.5 & & & \\
\hline
\end{tabular}


Table.2 Correlation between grain yield and yield attributing traits across the plant densities and genotypes of finger millet

\begin{tabular}{|c|c|c|c|c|c|c|c|c|c|c|c|}
\hline & (1) LAI & (2) LI & $\begin{array}{c}\text { (3) } \\
\text { Chlo. }\end{array}$ & $\begin{array}{c}\text { (4) } \\
\text { Photosy. }\end{array}$ & (5) PT & $\begin{array}{c}(6) \\
\text { MEW }\end{array}$ & (7) TW & $\begin{array}{c}(8) \\
\text { Spike }\end{array}$ & (9) HI & $\begin{array}{c}(\mathbf{1 0}) \\
\text { Biomass } \\
\end{array}$ & $\begin{array}{l}\text { (11) } \\
\text { GY }\end{array}$ \\
\hline (1) LAI & 1.000 & & & & & & & & & & \\
\hline (2) Light penetration & -0.637 & 1.000 & & & & & & & & & \\
\hline (3) Chlorosis & 0.556 & -0.507 & 1.000 & & & & & & & & \\
\hline (4) Photosynthetic rate & -0.317 & 0.381 & -0.337 & 1.000 & & & & & & & \\
\hline (5) Prod. Tillers $\mathrm{m}^{-2}$ & 0.687 & -0.677 & 0.875 & -0.505 & 1.000 & & & & & & \\
\hline (6) Mean ear weight & -0.642 & 0.668 & -0.836 & 0.439 & -0.967 & 1.000 & & & & & \\
\hline (7) Test weight & -0.188 & -0.349 & -0.315 & -0.195 & -0.210 & 0.180 & 1.000 & & & & \\
\hline (8) Spikelet fertility & -0.539 & 0.439 & -0.459 & 0.441 & -0.492 & 0.478 & -0.311 & 1.000 & & & \\
\hline (9) HI & -0.461 & 0.561 & -0.043 & 0.242 & -0.188 & 0.242 & -0.570 & 0.521 & 1.000 & & \\
\hline (10) Total biomass & 0.803 & -0.809 & 0.239 & -0.347 & 0.505 & -0.470 & 0.317 & -0.484 & -0.720 & 1.000 & \\
\hline (11) Grain yield & 0.528 & -0.457 & 0.356 & -0.217 & 0.536 & -0.424 & -0.269 & -0.034 & 0.277 & 0.457 & 1.000 \\
\hline
\end{tabular}

Note: $\mathrm{r}-$ value more than 0.433 and 0.549 are significant at 5 and $1 \%$ respectively 
Table.3 Effect of plant densities on leaf area index (LAI), light penetration and leaf chlorosis in finger millet genotypes

\begin{tabular}{|c|c|c|c|c|c|c|c|c|c|c|c|c|c|}
\hline \multirow[t]{2}{*}{$\begin{array}{l}\text { Spacing } \\
(\mathbf{c m} \times \mathbf{c m}) / \\
\text { Varieties }\end{array}$} & \multirow{2}{*}{$\begin{array}{c}\text { Plant } \\
\text { density } \\
(\mathrm{No} . \\
\left.\mathrm{m}^{-2}\right)\end{array}$} & \multicolumn{4}{|c|}{ LAI at flowering } & \multicolumn{4}{|c|}{$\begin{array}{c}\text { Light penetration }\left(\mu \mathrm{molm}^{-2} \mathrm{~s}^{-1}\right) \text { at } \\
\text { flowering }\end{array}$} & \multicolumn{4}{|c|}{$\begin{array}{c}\text { Leaf chlorosis at } 20 \text { DAF } \\
\text { (No. of chlorotic leaves per main } \\
\text { tiller) }\end{array}$} \\
\hline & & $\begin{array}{l}\text { GE- } \\
292\end{array}$ & $\begin{array}{l}\text { GE- } \\
199\end{array}$ & $\begin{array}{l}\text { GPU- } \\
28\end{array}$ & Mean & $\begin{array}{l}\text { GE- } \\
292\end{array}$ & $\begin{array}{l}\text { GE- } \\
199\end{array}$ & $\begin{array}{l}\text { GPU- } \\
28\end{array}$ & Mean & $\begin{array}{l}\text { GE- } \\
292\end{array}$ & $\begin{array}{l}\text { GE- } \\
199\end{array}$ & $\begin{array}{l}\text { GPU- } \\
28\end{array}$ & Mean \\
\hline$T_{1}(30 \times 15)$ & 22.2 & 4.61 & 4.17 & 5.18 & 4.65 & 146.5 & 163.9 & 44.3 & 118.2 & 0.11 & 0.11 & 0.00 & 0.07 \\
\hline$T_{2}(30 \times 10)$ & 33.3 & 5.65 & 4.25 & 5.80 & 5.23 & 115.6 & 175.4 & 53.4 & 114.8 & 0.00 & 0.00 & 0.00 & 0.00 \\
\hline$T_{3}(22.5 \times 10)$ & 44.4 & 5.76 & 6.04 & 7.96 & 6.58 & 60.9 & 69.6 & 18.1 & 49.5 & 0.33 & 0.11 & 0.00 & 0.15 \\
\hline$T_{4}(15 \times 10)$ & 66.7 & 9.27 & 6.88 & 6.34 & 7.50 & 42.3 & 62.4 & 31.8 & 45.5 & 0.56 & 0.44 & 0.22 & 0.41 \\
\hline$T_{5}(10 \times 10)$ & 100.0 & 8.64 & 7.25 & 5.67 & 7.19 & 35.2 & 48.4 & 19.0 & 34.2 & 1.33 & 1.22 & 0.89 & 1.15 \\
\hline$T_{6}(10 \times 7.5)$ & 133.3 & 8.82 & 5.85 & 6.18 & 6.95 & 34.7 & 42.0 & 22.4 & 33.0 & 1.89 & 1.33 & 1.22 & 1.48 \\
\hline$T_{7}(10 \times 5)$ & 200.0 & 8.81 & 6.37 & 6.41 & 7.20 & 33.7 & 39.6 & 15.1 & 29.5 & 2.56 & 1.89 & 1.56 & 2.00 \\
\hline Mean & & 7.37 & 5.83 & 6.22 & & 67.0 & 85.9 & 29.2 & & 0.97 & 0.73 & 0.56 & \\
\hline Factors & & $\mathrm{SEm} \pm$ & $\begin{array}{l}\text { C.D } \\
@ \mathrm{t} 5 \%\end{array}$ & & & $\mathrm{SEm} \pm$ & $\begin{array}{c}\mathrm{CD} @ \\
5 \%\end{array}$ & & & $\mathrm{SEm} \pm$ & $\begin{array}{c}\mathrm{CD} @ \\
5 \%\end{array}$ & & \\
\hline Treatments & & 0.20 & 0.58 & & & 3.1 & 9.0 & & & 0.09 & 0.26 & & \\
\hline Genotypes & & 0.13 & 0.38 & & & 2.0 & 5.8 & & & 0.06 & 0.16 & & \\
\hline Interaction & & 0.35 & 1.01 & & & 5.4 & 15.5 & & & 0.15 & NS & & \\
\hline C.V. $(5 \%)$ & & 9.4 & & & & 15.5 & & & & 35.8 & & & \\
\hline
\end{tabular}


Table.4 Effect of plant densities on photosynthetic rate and productive tillers in finger millet genotypes

\begin{tabular}{|c|c|c|c|c|c|c|c|c|c|c|c|c|c|}
\hline \multirow{2}{*}{$\begin{array}{l}\text { Spacing } \\
(\mathrm{cm} \times \mathrm{cm}) / \\
\text { Varieties }\end{array}$} & \multirow{2}{*}{$\begin{array}{c}\text { Plant } \\
\text { density } \\
(\mathrm{No} . \\
\left.\text { m }^{-2}\right)\end{array}$} & \multicolumn{4}{|c|}{ 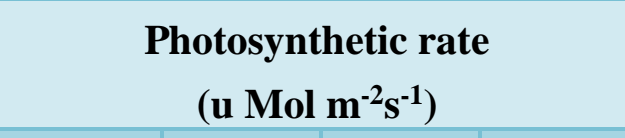 } & \multicolumn{4}{|c|}{ Productive tillers (No. $\mathbf{m}^{-2}$ ) } & \multicolumn{4}{|c|}{$\begin{array}{l}\text { Productive tillers } \\
\quad\left(\text { No. } \text { hill }^{-1}\right)\end{array}$} \\
\hline & & GE-292 & $\begin{array}{l}\text { GE- } \\
199\end{array}$ & $\begin{array}{l}\text { GPU- } \\
28\end{array}$ & Mean & $\begin{array}{l}\text { GE- } \\
292\end{array}$ & $\begin{array}{l}\text { GE- } \\
199\end{array}$ & $\begin{array}{l}\text { GPU- } \\
28\end{array}$ & Mean & $\begin{array}{l}\text { GE- } \\
292\end{array}$ & $\begin{array}{l}\text { GE- } \\
199\end{array}$ & $\begin{array}{l}\text { GPU- } \\
28\end{array}$ & Mean \\
\hline$T_{1}(30 \times 15)$ & 22.2 & 19.67 & 17.63 & 19.33 & 18.88 & 100.7 & 88.3 & 100.0 & 96.3 & 4.53 & 3.98 & 4.50 & 4.34 \\
\hline$T_{2}(30 \times 10)$ & 33.3 & 18.73 & 20.27 & 18.53 & 19.18 & 111.7 & 105.0 & 102.7 & 106.4 & 335 & 3.15 & 3.08 & 3.20 \\
\hline$T_{3}(22.5 \times 10)$ & 44.4 & 16.47 & 20.30 & 18.73 & 18.50 & 127.0 & 137.7 & 119.7 & 128.1 & 2.86 & 3.10 & 2.70 & 2.89 \\
\hline$T_{4}(15 \times 10)$ & 66.7 & 15.93 & 18.37 & 18.73 & 17.68 & 213.4 & 183.7 & 175.6 & 190.9 & 3.20 & 2.76 & 2.63 & 2.86 \\
\hline $\mathbf{T}_{5}(\mathbf{1 0} \times \mathbf{1 0})$ & 100.0 & 16.53 & 20.67 & 17.57 & 18.26 & 229.0 & 190.0 & 199.0 & 206.0 & 2.29 & 1.90 & 2.00 & 2.06 \\
\hline$T_{6}(10 \times 7.5)$ & 133.3 & 13.30 & 19.10 & 13.63 & 15.34 & 233.7 & 196.9 & 219.8 & 216.8 & 1.76 & 1.48 & 1.65 & 1.62 \\
\hline$T_{7}(10 \times 5)$ & 200.0 & 19.40 & 19.67 & 12.50 & 17.19 & 244.3 & 216.2 & 248.5 & 236.3 & 1.22 & 1.08 & 1.24 & 1.18 \\
\hline Mean & & 17.15 & 19.43 & 17.01 & & 180.0 & 159.7 & 166.5 & & 2.75 & 2.49 & 2.54 & \\
\hline Factors & & $\mathrm{SEm} \pm$ & $\begin{array}{c}\mathrm{CD} @ \\
5 \%\end{array}$ & & & $\mathrm{SEm} \pm$ & $\begin{array}{c}\mathrm{CD} @ \\
5 \%\end{array}$ & & & $\mathrm{SEm} \pm$ & $\begin{array}{c}\mathrm{CD} @ \\
5 \%\end{array}$ & & \\
\hline Treatments & & 1.43 & NS & & & 4.9 & 14.1 & & & 0.066 & 0.19 & & \\
\hline Genotypes & & 0.94 & NS & & & 3.2 & 9.2 & & & 0.043 & 0.12 & & \\
\hline Interaction & & 2.48 & NS & & & 8.5 & 24.4 & & & 0.115 & 0.33 & & \\
\hline C.V $(5 \%)$ & & 24.2 & & & & 8.7 & & & & 7.72 & & & \\
\hline
\end{tabular}


Table.5 Effect of plant densities on mean ear weight, test weight and spikelet fertility in finger millet genotypes

\begin{tabular}{|c|c|c|c|c|c|c|c|c|c|c|c|c|c|}
\hline \multirow{2}{*}{$\begin{array}{l}\text { Spacing } \\
(\mathrm{cm} \times \mathrm{cm}) / \\
\text { Varieties }\end{array}$} & \multirow{2}{*}{$\begin{array}{c}\text { Plants } \\
\text { (No. } \\
\text { m }^{-2} \text { ) }\end{array}$} & \multicolumn{4}{|c|}{ Mean ear weight (g) } & \multicolumn{4}{|c|}{ Test weight (g/ 1000 seeds) } & \multicolumn{4}{|c|}{ Spikelet fertility (\%) } \\
\hline & & $\begin{array}{l}\text { GE- } \\
292\end{array}$ & $\begin{array}{l}\text { GE- } \\
199\end{array}$ & $\begin{array}{c}\text { GPU- } \\
28\end{array}$ & Mean & $\begin{array}{l}\text { GE- } \\
292\end{array}$ & $\begin{array}{l}\text { GE- } \\
199\end{array}$ & $\begin{array}{c}\text { GPU- } \\
28\end{array}$ & Mean & $\begin{array}{l}\text { GE- } \\
292\end{array}$ & $\begin{array}{c}\text { GE- } \\
199\end{array}$ & $\begin{array}{c}\text { GPU- } \\
28\end{array}$ & Mean \\
\hline$T_{1}(30 \times 15)$ & 22.2 & 6.95 & 8.89 & 8.30 & 8.05 & 3.29 & 3.10 & 4.05 & 3.48 & 80.8 & 86.8 & 75.7 & 81.1 \\
\hline$T_{2}(30 \times 10)$ & 33.3 & 6.92 & 7.61 & 7.10 & 7.21 & 3.25 & 3.12 & 4.11 & 3.49 & 82.1 & 76.6 & 74.9 & $\mathbf{7 7 . 8}$ \\
\hline$T_{3}(22.5 \times 10)$ & 44.4 & 6.83 & 7.21 & 7.20 & 7.08 & 3.27 & 2.99 & 3.86 & 3.37 & 77.0 & 93.0 & 73.6 & 81.2 \\
\hline$T_{4}(15 \times 10)$ & 66.7 & 4.56 & 5.28 & 5.07 & 4.97 & 3.10 & 3.20 & 3.71 & 3.34 & 74.4 & 80.6 & 78.4 & $\mathbf{7 7 . 8}$ \\
\hline$T_{5}(10 \times 10)$ & 100.0 & 3.62 & 4.80 & 4.20 & 4.21 & 3.08 & 3.28 & 3.68 & 3.35 & 64.4 & 76.8 & 75.9 & 72.4 \\
\hline$T_{6}(10 \times 7.5)$ & 133.3 & 3.94 & 4.27 & 4.10 & 4.11 & 3.05 & 2.97 & 3.84 & 3.29 & 74.5 & 87.0 & 75.5 & 79.0 \\
\hline$T_{7}(10 \times 5)$ & 200.0 & 3.74 & 4.17 & 3.75 & 3.89 & 3.07 & 2.99 & 3.82 & 3.29 & 64.3 & 81.5 & 65.1 & 70.3 \\
\hline Mean & & 5.23 & 6.04 & 5.67 & & 3.16 & 3.09 & 3.87 & & 73.9 & 83.2 & 74.1 & \\
\hline Factors & & SEm \pm & $\begin{array}{l}\text { C.D } \\
@ \text { ! } 5 \%\end{array}$ & & & SEm \pm & $\begin{array}{c}\text { CD @ } \\
5 \%\end{array}$ & & & SEm \pm & $\begin{array}{c}\text { CD @ } \\
5 \%\end{array}$ & & \\
\hline Treatments & & 0.22 & 0.63 & & & 0.054 & 0.155 & & & 1.77 & 5.1 & & \\
\hline Genotypes & & 0.14 & 0.41 & & & 0.035 & 0.101 & & & 1.16 & 3.31 & & \\
\hline Interaction & & 0.38 & NS & & & 0.094 & NS & & & 3.07 & 8.8 & & \\
\hline C.V (5\%) & & 11.6 & & & & 4.8 & & & & 6.89 & & & \\
\hline
\end{tabular}



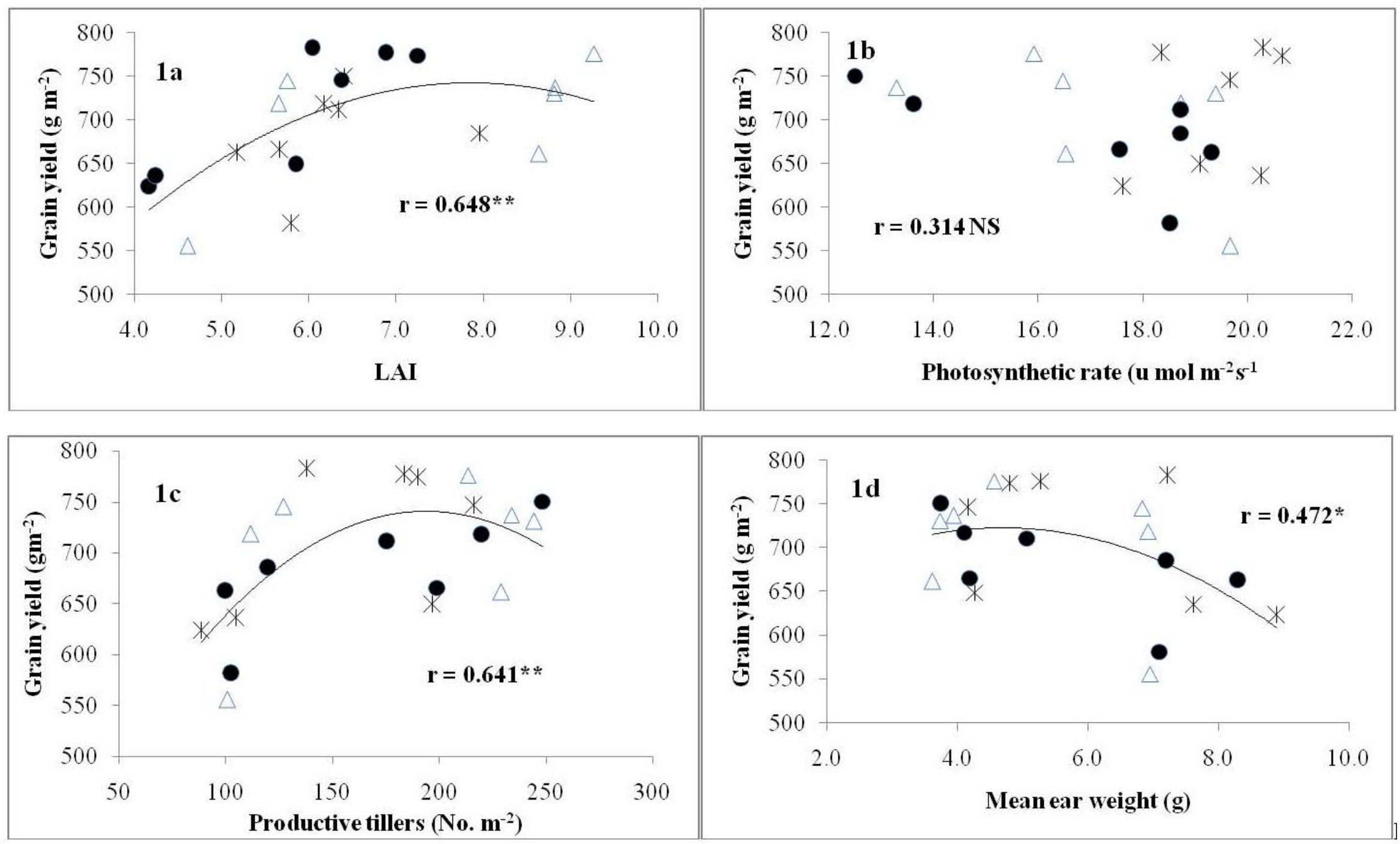

Fig.1 Relationship between source size, yield parameters and grain yield in finger millet genotypes

$$
(*=\mathrm{GE}-199, \Delta=\mathrm{GE}-292, \bullet=\mathrm{GPU}-28)
$$


The wider spacing also reduce the productive tiller number per unit land area significantly and thus decreased grain yield (Table 4 and Anitha, 2015; Nigus and Melese, 2018). The results reiterate that the source is not a major limitation under optimal irrigation conditions in finger millet.

Another important trait that determines the biomass production and grain yield is the source activity (photosynthetic rate). The photosynthetic rate did not differ significantly between the planting densities or varieties (Table 4). Photosynthetic rate was not related significantly to biomass and grain yield (Table 2 and Fig. 1b). The finger millet being $\mathrm{C}_{4}$ (NAD-ME) species (Ueno et al., 2006) has higher photosynthetic rate and thus the photosynthetic rate is not a limitation, rather light interception by the lower leaves at narrow spacing is a major constraint. Therefore, possible suggestions for yield improvement under optimal irrigation conditions could be through selection and breeding for leaf acute angle to result in higher light use efficiency as source is not a limitation.

The study show that, the source size (LAI) and source activity (photosynthetic rate) in finger millet (GPU-28) is not a limitation under optimal input conditions, but the sink parameters such as productive tillers or ear size could be the limitations for higher productivity (Bezaweletaw et al., 2006; Assefa et al., 2013; Dineshkumar et al., 2014; Maobe et al., 2014; Jadhav et al., 2015; Madhavilatha and Subbarao, 2015; Simbagije, 2016). The productive tillers $\mathrm{m}^{-2}$ (sink number) was significantly increased with increased plant density from $44.4 \mathrm{~m}^{-2}$ and above (Table 4) but the grain yield was not increased significantly although the relationship between productive tillers and grain yield was significantly positive (Table 2; Fig. 1c). In contrast, a negative correlation between the tiller number and grain yield has been reported due to significant decrease in ear size (Jyothsna et al., 2016). The mean ear weight (sink size) was related to grain yield positively and significantly (Fig. 1d) but beyond $5 \mathrm{~g} \mathrm{ear}^{-1}$, the yield was in declining trend, this clearly suggests the compensation mechanism between tiller number and ear size $\left(\mathrm{r}=0.998^{* *}\right.$, Table 2 and 5). In addition, increased plant density above $33.3 \mathrm{~m}^{-2}$ decreased the test weight (Table 5). With respect to spikelet fertility, although particular trend is not observed, at closer spacing (high plant density), the spikelet fertility was markedly low (Table 5).

Increase in tiller number per unit land area (above 44.4 hills $\mathrm{m}^{-2}$ ) by reduced spacing, will lead to management problems like weed management and disease management (Bitew and Asargew, 2014) with no significant increase in grain yield. Therefore spacing of $22.5 \mathrm{~cm} \times 10 \mathrm{~cm}$ could be optimum. Other research reports also show that $25 \mathrm{~cm} \times 25 \mathrm{~cm}$ over the $10 \mathrm{~cm} \times 10 \mathrm{~cm}$ (Bhatta et al., 2017) and $20 \mathrm{~cm}$ between the rows as over the 10 $\mathrm{cm}$ gave better grain yields (Shinggu and Gani, 2012).

At the spacing of $22.5 \mathrm{~cm} \mathrm{x} 10 \mathrm{~cm}$, increase in productive tiller number or ear size can increase the grain yield as source is not a constraint in finger millet. In this direction, Kalpana et al., (2016) reported that at a given spacing, increase in tiller number per hill up to 4.9 increased the grain yield of finger millet. Therefore, further improvement in grain yield of finger millet could be possible by (i) increased productive tillers per hill to five at the spacing of $22.5 \mathrm{~cm} \mathrm{x} 10 \mathrm{~cm}$ or 30 $\mathrm{cm} \times 7.5 \mathrm{~cm}$ by management practices (Damar et al., 2016), (ii) identifying genotypes with erect leaves to intercept more sunlight, (iii) removal of old leaves which acts as sink during reproductive phase and planting two seedlings per hill. 


\section{Acknowledgments}

Authors thank Dr. S. Ramesh, Professor, Department of Plant Breeding \& Genetics, Dr. H.M. Jayadeva, Professor, Department of Agronomy for their suggestions and support to conduct the experiment.

\section{References}

Anitha, D., 2015. Finger millet (Eleusine coracana (L.) Gaertn.) productivity as influenced by planting geometry and age of seedlings. M.Sc. (Agri.) Thesis, ANGRAU, Hyderabad.

Anonymous, 2015. Annual Report 2014-15, All India Coordinated Small millets Improvement Project, GKVK, Bengaluru. Ag., Pp. 1.

Assefa, A., Fetene, M. and Tesfaye, K., 2013. Agro-morphological, physiological and yield related performances of finger millet [Eleusine coracana (1.) Gaertn.] accessions evaluated for drought resistance under field condition. Asian Journal of Agriculture and Rural Development. 3(10): 709-720.

Berdahl, J. D, Rasmusson, D. C. and Moss, D. N., 1971. Effects of leaf area on photosynthetic rate, light penetration and grain yield in Barley. American Society of Agronomy. 12 (2): 177 - 180.

Bezaweletaw, K., Sripichitt, P., Wongyai, W. and Hongtrakul, V., 2006. Genetic variation, heritability and path analysis in Ethiopian finger millet land races. Kasetsart Journal of Natural Science. 40: 322-334.

Bhatta, L. R., Subedi, R., Joshi, P. and Gurung, S. B., 2017. Effect of crop establishment methods and varieties on tillering habit, growth rate and yield of finger millet. Agriculture Research and Technology. 11(5): 555826.

Bitew, Y. and Asargew, F., 2014. Determination of seed rate and inter row spacing for finger millet production (Eleusine coracana (L.) Gaertn.)in North Western Ethiopia. International Journal of Research and Review. 1(4): 1-7

Chandra, D., Chandra, S., Pallavi, and Sharma, A. K., 2016. Review of finger millet (Eleusine coracana (L.) Gaertn): A power house of health benefiting nutrients. Food Science and Human Wellness. 5: 149 - 155.

Chavan, B.R., Jawale, L.N. and Shinde, A.V., 2020. Correlation and path analysis studies in finger millet for yield and yield contributing traits (Eleusine coracana L. Gaertn). International Journal of Chemical Studies. 8(1): 2911-2914.

Damar, W.K., Garba, A., Russom, Z., Ibrahim, S. A., Haggai, P.T. and Dikwahal, H.D., 2016. Effect of poultry manure on growth and yield of finger millet (Eleusine coracana $\mathrm{L}$. Gaertn) in the northern Guinea Savannah, Nigeria, Production Agriculture and Technology. 12 (1): 173-180.

Davis, K.F., Chhtre, A., Rao, N.D., Singh, D. and DeFries, R., 2019. Sensitivity of grain yields to historical climate variability in India. Environmental Research Letters, 14: 064013, http://doi.org/10.1088/17489326/ab22db.

Dereje, G., Adisu, T. and Anbessa, B., 2016. Influence of row spacing and seed rate on yield and yield components of finger millet at Assosa zone in Benshagul Gumuze region of Ethiopia. Journal of Biology, Agriculture and Healthcare. 6(5): 42-45.

Devi, P.B., Vijayabharathi, R., Sathyabama, S., Malleshi, N.G. and Priyadarisini,V.B., 2014. Health benefits of finger millet (Eleusine coracana L.) polyphenols and dietary fiber: A review. Journal of Food Science and Technology. 51: 1021-1040.

Dida, M. M., Srinivasachary, Ramakrishnan, S., Bennetzen, J.L., Gale, M.D. and Devos, K.M., 2007. The genetic map of finger millet (Eleusine coracana L.). Theoretical and Applied Genetics. 114: 321-332.

Dineshkumar, Tyagi, V. and Ramesh, B., 2014. Path coefficient analysis for yield and its contributing traits in finger millet. International Journal of Advanced Research. 2 (8): 235-240.

Gull, A., Jan, R., Nayik, G.A., Prasad, K. and Kumar, P., 2014. Significance of finger millet in nutrition, health and value added products: A review. Journal of Environmental Science and Computer Science, Engineering Technology. 3: 16011608. 
Gupta, S.M., Arora, S., Mirza, N., Pande, A., Lata, C. and Puranik, S., 2017. Finger millet: a "certain" crop for an "uncertain" future and a solution to food insecurity and hidden hunger under stressful environments. Frontiers in Plant Science. 8: 643, doi: 10.3389/fpls.2017.00643

Jadhav, R., Ratna Babu, D., Lal Ahamed, M. and Srinivasa Rao, V., 2015. Character association and path coefficient analysis for grain yield and yield components in finger millet (Eleusine coracana (L.) Gaertn.). Electronic Journal of Plant Breeding. 6(2): 535-539.

Jyothsna, S., Patro, T.S.S.K., Ashok, S., Sandhya Rani, Y. and Neeraja,B., 2016. Studies on genetic parameters, character association and path analysis of yield and its components in finger millet (Eluesine Coracana L. Gaertn), International Journal of Theoretical and Applied Sciences. 8(1): 25-30.

Kalpana, K., Vadivel1, N., Bharathikumar, K and Kavimani, R., 2016. Seasonal influence on the occurrence and management of blast of finger millet (Eleusine coracana (1.) Gaertn.) under field condition. International Journal of Advances in Agricultural Science and Technology. 3(1): 43-51.

Krishnamurthy, K., 1971. Response of finger millet varieties under various levelsof nitrogen. Annuals of Arid Zone. 10: 261265.

Kumari, P.L. and Sumathi, S., 2002. Effect of consumption of finger millet on hyperglycemia in non-insulin dependent diabetes mellitus (NIDDM) subjects. Plant Foods for Human Nutrition. 57(3-4): 205213.

Madhavilatha, L. and SubbaRao, M., 2015. Performance of elite finger millet cultures for grain yield, yield influencing traits and blast tolerance, International Journal of Agricultural Science and Research. 5 (1): 111-114.

Maobe, S.N., Nyang'au, M.K., Basweti, E.A., Getabu, A., Mwangi, T.J. and Ondicho, A.R., 2014. Effect of plant density on growth and grain yield of finger millet (Eleusine coracana) under high potential conditions of Southwest Kenya. World Journal of Agricultural Sciences. 10(6): 261-268.

Nanja Reddy, Y.A., Jayarame Gowda, Ashok, E.G., Krishne Gowda, K.T. and Gowda, M.V.C., 2019. Higher leaf area improves the productivity of finger millet (Eleusine coracana (L.) Gaertn.) under rainfed conditions. Intentional Journal of Current Microbiology and Applied Sciences. 8(5): 1369-1377.

Negi, S., Bhatt, A. and Kumar, V., 2017. Character association and path analysis for yield and its related traits in finger millet (Eleusine coracana (L.) Gaertn) genotypes. Journal of Applied and Natural Science. 9(3): 1624-1629.

Nigus, C. and Melese, B., 2018. Inter row spacing and seed rate effect on finger millet (Eleusine coracana (L.) production at MerbLekhe District, Ethiopia. Journal of Agricultural Research. 3(4): 000169.

Patrick, J.W., 1988. Assimilate partitioning in relation to crop productivity. Hort Science. 23: 33-40.

Prakasha, G., Kalyana Murthy, K.N., Prathima, A.S. and Meti, R.N., 2018. Effect of spacing and nutrient levels on growth attributes and yield of finger millet (Eleusine coracana L. Gaertn.) cultivated under Guni planting method in red sandy loamy soil of Karnataka, India. International Journal of Current Microbiology and Applied Sciences. 7(05): 1337-1343.

Ramakrishnan, M., Ceasar, S.A., Vinod, K., Duraipandiyan, V., Krishna, T. A. and Upadhyaya, H.D., 2017. Identification of putative QTLs for seedling stage phosphorus starvation response in finger millet (Eleusine coracana L. Gaertn.) by association mapping and cross species synteny analysis. PLoS One.12:e0183261.

Richards. R. A., 2000. Selectable traits to increase crop photosynthesis and yield of grain crops. Journal of Experimental Botany. 51: 447 - 458.

Sakamma, S., Umesh, K.B., Girish, M.R., Ravi, S.C., Satishkumar, M. and Bellundagi, V., 2018. Finger millet (Eleusine coracana L. Gaertn.) production system: status, 
potential, constraints and implications for improving small farmer's welfare. Journal of Agricultural Sciences. 10: 162-179.

Sharma, D., Jamra, G., Singh, U.M., Sood, S. and Kumar, A., 2017. Calcium bio-fortification: three pronged molecular approaches for dissecting complex trait of calcium nutrition in finger millet (Eleusine coracana) for devising strategies of enrichment of food crops. Frontiers in Plant Science. 7: 2028.doi: 10.3389/fpls.2016.02028

Sheoran, O. P., Tonk, D. S., Kaushik, L. S., Hasija, R. C. and Pannu, R. S., 1998. Statistical software package for agricultural research workers. Recent advances in information theory, statistics \& computer applications, Department of Mathematics Statistics, CCS HAU, Hisar, 139-143

Shinggu, C.P. and Gani, M, 2012. Effects of planting methods, sowing dates and spacing on weed and the productivity of finger millet (Eleusine corocana L. Gaertn.) in the northern guinea Savanna of Nigeria. Global Journal of Bioscience and Biotechnology. 1(2): 160-162.

Shobana, S., Krishnaswamy, K., Sudha, V., Malleshi, N.G., Anjana, R.M., Palaniappan, L. and Mohan, V., 2013. Finger millet (Ragi, Eleusine coracana L.): A review of its nutritional properties, processing, and plausible health benefits. Advances in Food Nutrition Research. 69: 1-39.

Simbagije, R.M., 2016. Diversity of finger millet (Eleusine coracana (L.) Gaertn.) genotypes on drought tolerance and yield in Tanzania, Ph.D. Thesis submitted to Sokoine
University of Agriculture. Morogoro, Tanzania.

Somashekhar and Loganandhan, N., 2020. SRIfinger millet cultivation: A case Study in Tumakuru District, India. International Journal of Current Microbiology and Applied Sciences, 9(01): 2089-2094.

Swetha, T. N., 2011. Assessment of the contribution of physiological traits to grain yield during crop improvement of finger millet (Eleusine coracana L. Gaertn.). M.Sc. (Agri.) Thesis, Univ. Agric. Sci., Bangalore.

Thilakarathna, M.S. and Raizada, M.N., 2015. A review of nutrient management studies involving finger millet in the semi-arid tropics of Asia and Africa. Agronomy. 5: 262-290.

Ueno, O., Kawano, Y., Wakayama, M. and Takeda, T., 2006. Leaf vascular systems in $\mathrm{C}_{3}$ and $\mathrm{C}_{4}$ Grasses: A two-dimensional analysis. Annals of Botany. 97(4): 611 621.

Wafula, W.N., Korir, N.K., Ojulong, H.F., Siambi, M. and Gweyi-Onyango, J.P., 2018. Protein, calcium, zinc, and iron contents of finger millet grain response to varietal differences and phosphorus application in Kenya. Agronomy. 8: 24; doi: 10.3390

Yoseph, T., 2014. Determination of Inter Row Spacing and Seed Rate on Productivity of Finger Millet [Eleusine Coracana (L.) Gaertn.], At Jinka, Southern Ethiopia. International Journal of Research in Agricultural Sciences, 1(3): 172-176.

\section{How to cite this article:}

Mujahid Anjum, Y. A. Nanja Reddy and Sheshshayee. M. S. 2020. Optimum LAI for Yield Maximisation of Finger Millet under Irrigated Conditions. Int.J.Curr.Microbiol.App.Sci. 9(05): 1535-1547. doi: https://doi.org/10.20546/ijcmas.2020.905.174 